\title{
Elevated serum urate is a potential factor in reduction of total bilirubin: a Mendelian randomization study
}

Hui Zhang ${ }^{1, *}$, Jing Liu' ${ }^{1, *}$, Zheng Dong ${ }^{1, *}$, Yue Ding ${ }^{2}$, Qiaoxia Qian ${ }^{2}$, Jingru Zhou ${ }^{2}$, Yanyun Ma ${ }^{2}$, Zhendong Mei ${ }^{1}$, Xiangxiang Chen ${ }^{1}$, Yuan Li ${ }^{1}$, Ziyu Yuan ${ }^{3}$, Juan Zhang ${ }^{3}$, Yajun Yang ${ }^{2,3}$, Xingdong Chen ${ }^{1,3}$, Li Jin ${ }^{1,3}$, Hejian Zou ${ }^{4,5}$, Xiaofeng Wang ${ }^{1,3}$ and Jiucun Wang ${ }^{1,3,5}$

${ }^{1}$ State Key Laboratory of Genetic Engineering, Collaborative Innovation Center for Genetics and Development, School of Life Sciences, Fudan University, Shanghai, China

${ }^{2}$ Ministry of Education Key Laboratory of Contemporary Anthropology, School of Life Sciences, Fudan University, Shanghai, China

${ }^{3}$ Fudan-Taizhou Institute of Health Sciences, Taizhou, Jiangsu, China

${ }^{4}$ Division of Rheumatology, Huashan Hospital, Fudan University, Shanghai, China

${ }^{5}$ Institute of Rheumatology, Immunology and Allergy, Fudan University, Shanghai, China

"These authors contributed equally to this work

Correspondence to: Jiucun Wang, email: jcwang@fudan.edu.cn Xiaofeng Wang, email: xiaofengwang@fudan.edu.cn

Keywords: serum urate, total bilirubin, mendelian randomization study

Received: August 29, 2016 Accepted: October 03, $2017 \quad$ Published: October 24, 2017

Copyright: Zhang et al. This is an open-access article distributed under the terms of the Creative Commons Attribution License 3.0 (CC BY 3.0), which permits unrestricted use, distribution, and reproduction in any medium, provided the original author and source are credited.

\section{ABSTRACT}

Aim: A Mendelian randomization study (MRS) can be linked to a "natural" randomized controlled trial in order to avoid potential bias of observational epidemiology. We aimed to study the possible association between serum urate (SU) and total bilirubin (TBIL) using MRS.

Materials and Methods: An observational epidemiological study using ordinary least squares (OLS) regression and MRS using two-stage least square (TLS) regression was conducted to assess the effect of SU on TBIL. The comparison between the OLS regression and the TLS regression was analyzed by the Durbin-Hausman test. If the $p$ value is significant, it suggests that the OLS regression cannot evaluate the relationship between exposure and outcome, and the TLS regression is precise; while if the $p$ value is not significant, there would be no significant difference between the two regressions.

Results: A total of 3,753 subjects were analyzed. In OLS regression, there was no significant association between SU and TBIL in all subjects and subgroup analysis (all $p>0.05$ ). However, MRS revealed a negative correlation between SU and TBIL after adjustment for confounders (beta $=-0.021, p=0.010$ ). Further analysis was conducted in different SU subgroups, and results show that elevated SU was associated with a significant reduction in TBIL after adjustment for hyperuricemic subjects (beta $=-0.053, p=0.027$ ). In addition, the results using the DurbinHausman test further confirmed a negative effect of SU on TBIL $(p=0.002$ and 0.010 , respectively).

Conclusions: This research shows for the first time that elevated SU was a potential causal factor in the reduction of TBIL and it provides strong evidence to resolve the controversial association between SU and TBIL. 


\section{INTRODUCTION}

Uric acid is the end-product of purine metabolism in humans and is controlled by several genetic factors [1], such as $A B C G 2$ and $S L C 2 A 9$ [2]. It has been reported that hyperuricemia can be one of the factors inducing oxidative stress followed by a pro-inflammatory process and endocrine dysfunction in adipose tissue [3]. Bilirubin had powerful antioxidant properties [4-6] and could be oxidized to biliverdin by reactive oxygen species (ROS) which could then protect cells from oxidative stress [7]. Observational studies about the relationship between serum urate (SU) and total bilirubin (TBIL) levels were controversial. An observational study conducted in Korea has suggested that SU positively correlates with TBIL levels [8]. However, two reports found no association between SU and TBIL in Korea and Japan $[9,10]$. Surprisingly, a researcher in Serbia found that SU were positively associated with TBIL in men, but not in women [11]. It was generally known that the results from conventional observational epidemiology were more likely misinterpreted by a variety of confounders, such as lifestyle, socioeconomic factors, baseline health status, reverse causation, selected bias or other potentially unknown factors [12]. For example, SU levels were strongly affected by hormonal factors [13], body composition [14], diuretic use [15] and son on. Therefore, it was difficult to confirm whether SU was a real mediator of TBIL or whether the observed relationship between SU and TBIL was attributable to confounders. Therefore, it is difficult to confirm whether SU is a real causal of TBIL or whether the observed relationship between SU and TBIL was attributable to confounders.

The Mendelian randomization study (MRS) has been linked to a "natural" randomized controlled trial $[16,17]$. It is an approach to identify genetic variants (instrumental variables) that represent the intermediate phenotype (serum uric acid in this study) and the effect of the instrument on outcome (TBIL in this study). Therefore, it can be used to study whether an intermediate phenotype has a causal effect on outcome [18]. A study with genetic variants employed as instrumental variable could avoid potential bias of observational epidemiology that comes from confounders and reverse causation $[19,20]$. MRS has developed and progressed methodologically and increasingly applied in epidemiology to measure the causal relationship between exposures and outcomes in recent years. In addition, it has been gradually expanding the landscape of causal relationships in non-communicable chronic diseases [21]. In addition, the combined information of multiple variants as instruments in the study would increase statistical power to test the relationship between exposures and outcomes [22-24] and would avoid potential bias caused by weak instruments [25].

In this study, we used the MRS with genetic variants as the instrumental variable to investigate the potentially causal relationship between SU and TBIL levels.

\section{RESULTS}

\section{The reliability of the instrumental variable}

A total of 3,753 subjects, including 2,713 males and 1,040 females, were recruited in this study. The mean age of the population was $69.43 \pm 8.96$ years (Table 1). The total genetic risk score was selected as the instrumental variable in the Mendelian randomization analysis. There was a robust association between the total genetic risk score and SU; the F-statistic was 119.00. In addition, $\mathrm{R}^{2}$ represents the variance in $\mathrm{SU}$ explained by the instrumental variable, and the total genetic risk score explained $3.1 \%$ of the variance in $\mathrm{SU}$ in our population (Table 2). Furthermore, the other biochemical variables and the relationship between total genetic risk score and potential confounders are shown in Table 3. There was no relationship between total genetic risk score and confounders with $p$ values greater than 0.05 after adjustment (age: $p=0.086$; gender: $p=0.090$; BMI: $p=0.573$; FBG: $p=0.765$; BUN: $p=0.765$; respectively), suggesting no evidence for relationship between the total genetic risk score and other factors. Therefore, the selected instrumental variables in our MRS were adequate.

\section{Elevated serum urate was a potential causal factor in reduction of TBIL}

The results of conventional epidemiological analysis by the OLS method and Mendelian randomization analysis with the TLS approach are shown in Table 4. In the OLS regression, there was no significant relationship between SU and TBIL (for males: beta $=-0.003 ; p=0.061$; for females: beta $=0.002, p=0.201$; respectively); after adjusting for age, BMI, BUN and FBG, similar results were found between SU and TBIL in gender subgroups (male: beta $=-0.001, p=0.589$; female: beta $=0.002, p=$ 0.242 ; respectively). However, using the TLS approach, a negative correlation between SU and TBIL was identified (beta $=-0.021, p=0.009$ ). In gender subgroup analysis, the negative correlation was significant in males (beta $=$ $-0.020, p=0.035$ ) but not in females (beta $=-0.006, p=$ $0.491)$. Similar results were also observed after adjustment for confounders (for all: beta $=-0.021, p=0.010$; for males: beta $=-0.021, p=0.037$; for females: beta $=$ $-0.006, p=0.491)$. In addition, the comparison between the OLS analysis and the TLS analysis was calculated, and a significant difference was found in the adjusted analysis (for all: $\mathrm{DHp}=0.002$; for males: $\mathrm{DHp}=0.038$; for females: $\mathrm{DHp}=0.320$ ). Therefore, these results suggest that elevated serum urate was a potential causal factor in reduction of TBIL. 
Table 1: Summary of the characteristics of the subjects

\begin{tabular}{|c|c|c|c|c|c|c|}
\hline & \multicolumn{2}{|c|}{ All $(3,753)$} & \multicolumn{2}{|c|}{ Males $(2,713)$} & \multicolumn{2}{|c|}{ Females $(1,040)$} \\
\hline & Mean & SD & Mean & SD & Mean & SD \\
\hline Age (years) & 69.43 & 8.96 & 70.49 & 8.96 & 66.69 & 8.36 \\
\hline BMI (kg/m2) & 24.80 & 3.38 & 24.67 & 3.26 & 25.13 & 3.67 \\
\hline BUN (mmol/L) & 5.65 & 2.10 & 5.78 & 2.29 & 5.33 & 1.44 \\
\hline $\mathrm{FBG}(\mathrm{mmol} / \mathrm{L})$ & 5.59 & 1.61 & 5.60 & 1.64 & 5.59 & 1.54 \\
\hline TBIL $(\mu \mathrm{mol} / \mathrm{L})$ & 19.25 & 8.21 & 20.11 & 8.62 & 17.01 & 6.56 \\
\hline $\mathrm{SU}(\mu \mathrm{mol} / \mathrm{L})$ & 347.24 & 101.07 & 360.27 & 95.61 & 312.21 & 106.45 \\
\hline
\end{tabular}

BMI: Body mass index; BUN: Blood urea nitrogen; FBG: Fasting blood glucose; TBIL: Total bilirubin; SU: Serum urate; SD: Standard deviation

Table 2: Association between uric acid transporter genetic risk score and SU

\begin{tabular}{lccc}
\hline & F-statistic & $\mathbf{R}^{2}$ & $p$ \\
\hline Overall & 119.00 & 0.031 & $<0.001$ \\
Males & 127.50 & 0.045 & $<0.001$ \\
Females & 69.12 & 0.062 & $<0.001$ \\
\hline
\end{tabular}

The F-statistic represents the strength between the instrumental variables and UA; $\mathrm{R}^{2}$ represents the variance in SU explained by the instrumental variables.

\section{Elevated serum urate was a potential causal factor in reduction of TBIL in individuals with hyperuricemia}

Further studies were conducted in normal and hyperuricemia subgroups (Table 5). In the normal SU subgroup, a significant association between SU and TBIL was found by the OLS regression (for crude: beta $=0.013, p<$ 0.001 ; for adjusted: beta $=0.011, p<0.001)$. However, when stratified by gender, no significant association was found in either males or females $(p>0.05)$. In the TLS regression analysis, there was no significant association between SU and TBIL (for crude: beta $=-0.047, p=0.109$; for adjusted: beta $=-0.047, p=0.092$ ), and the $\mathrm{DH} p$ value was 0.025 and 0.002 in crude and adjusted analysis respectively, suggesting the result of OLS regression was affected by potential confounders or reverse causality, because it was different from the results of the TLS regression. Moreover, no association was observed between SU and TBIL in males (for crude: beta $=0.006, p=0.085$; for adjusted: beta $=0.007$, $p=0.063$ ) and females (for crude: beta $=0.018, p=0.477$; for adjusted: beta $=0.010, p=0.698)$.

In addition, the OLS and TLS regression was also performed in hyperuricemia subjects (Table 5). In the OLS regression, no relationship between SU and TBIL was found in both crude (for all: beta $=-0.003, p$ $=0.441$; for male: beta $=-0.007, p=0.141$; for female: beta $=-0.007, p=0.141$ ) and adjusted analysis (for all: beta $=0.001, p=0.854$; for male: beta $=-0.003, p=$ 0.523 ; for female: beta $=0.010, p=0.147)$. In the TLS regression analysis, there was no significant relationship between SU and TBIL in crude analysis (beta $=-0.053$, $p=0.053$ ), but after adjusting for confounders, increased SU was associated with significant reduction of TBIL in hyperuricemia individuals (beta $=-0.053, p=0.027$ ); the Durbin-Hausman test provided further evidence of a negative effect of SU on TBIL (DHp $=0.010)$. Similarly, further analysis was conducted in gender subgroups, and significant association was found in males (beta $=-0.056, p=0.049$ ) but not in females (beta $=-0.107$, $p=0.240)$ after adjustment for age, BMI, BUN and FBG. The Durbin-Hausman test provided further evidence of a negative effect of SU on TBIL (DHp $=0.039$ and 0.050 , respectively).

\section{DISCUSSION}

The theoretical foundation of Mendelian randomization depends on three assumptions [12, 26, 27]. (I) The instrumental variable is robustly associated with modifiable exposure of interest, with the F-statistic being considerably greater than ten [28]. In this study, the F-statistic was 119.00 and the $\mathrm{R}^{2}$ was 0.031 (Table 2), indicating a robust relationship between the genetic risk score (instrumental variable) and SU (exposure of interest). (II) The instrumental variables are not associated with confounding factors that cause biased conventional epidemiological associations between modifiable risk factors and outcomes. This assumption could be examined by verifying the instrumental variable for association by multivariate linear regression with adjusted for confounders (Table 3 ). There was no evidence 


\begin{tabular}{|c|c|c|c|c|}
\hline & & beta & SE & $P$ \\
\hline \multicolumn{5}{|l|}{ Age } \\
\hline \multirow[t]{2}{*}{ All } & Crude & -0.105 & 0.047 & 0.027 \\
\hline & Adjusted & -0.084 & 0.049 & 0.086 \\
\hline \multirow[t]{2}{*}{ Males } & Crude & -0.126 & 0.051 & 0.012 \\
\hline & Adjusted & -0.112 & 5.655 & 0.037 \\
\hline \multirow[t]{2}{*}{ Females } & Crude & 0.083 & 0.111 & 0.455 \\
\hline & Adjusted & 0.055 & 0.113 & 0.625 \\
\hline \multicolumn{5}{|l|}{ FBG } \\
\hline \multirow[t]{2}{*}{ All } & Crude & 0.194 & 0.263 & 0.459 \\
\hline & Adjusted & 0.150 & 0.266 & 0.573 \\
\hline \multirow[t]{2}{*}{ Males } & Crude & -0.246 & 0.278 & 0.376 \\
\hline & Adjusted & -0.262 & 0.279 & 0.349 \\
\hline \multirow[t]{2}{*}{ Females } & Crude & 1.109 & 0.600 & 0.065 \\
\hline & Adjusted & 0.540 & 0.624 & 0.387 \\
\hline \multicolumn{5}{|l|}{ BUN } \\
\hline \multirow[t]{2}{*}{ All } & Crude & -0.003 & 0.202 & 0.986 \\
\hline & Adjusted & 0.061 & 0.203 & 0.765 \\
\hline \multirow[t]{2}{*}{ Males } & Crude & 0.028 & 0.198 & 0.886 \\
\hline & Adjusted & 0.065 & 0.200 & 0.746 \\
\hline \multirow[t]{2}{*}{ Females } & Crude & 1.084 & 0.640 & 0.094 \\
\hline & Adjusted & 0.965 & 0.655 & 0.141 \\
\hline \multicolumn{5}{|l|}{ BMI } \\
\hline \multirow[t]{2}{*}{ All } & Crude & 0.149 & 0.126 & 0.235 \\
\hline & Adjusted & 0.102 & 0.203 & 0.765 \\
\hline \multirow[t]{2}{*}{ Males } & Crude & 0.223 & 0.140 & 0.112 \\
\hline & Adjusted & 0.182 & 0.143 & 0.199 \\
\hline \multirow[t]{2}{*}{ Females } & Crude & 0.269 & 0.252 & 0.285 \\
\hline & Adjusted & 0.206 & 0254 & 0.417 \\
\hline
\end{tabular}

Each independent test variable was adjusted by the other three variables (gender has been adjusted in All group). SE: Standard error; BMI: Body mass index; BUN: Blood urea nitrogen; FBG: Fasting blood glucose; SE: Standard error.

for a relationship between the uric acid transporter as the instrumental variable and any other confounders tested, all $p$ values were greater than 0.05 (age, gender, BMI, FBG and BUN). (III) The instrumental variables are related to the outcome only via its association with the modifiable exposure. This assumption was difficult to assess, and should be considered Despite this, the possibility of pleiotropic effects of the uric transporter instrumental variable on TBIL levels was difficult to exclude [29]. We did note that some of the uric acid transporters (particularly $A B C G 2$ [30]) were widely expressed and may have pleiotropic effects (i.e. effects on TBIL levels aside from or in addition to a direct effect of the serum urate). To reduce the possibility of violating the assumption, the Mendelian randomization approach was adjusted by age, gender, BMI, FBG and BUN in our study. Therefore, we were able to show that the selected instrumental variables in our MRS were adequate.

This study investigated for the first time the association between SU and TBIL levels using MRS. The Mendelian randomization employed the TLS approach with the uric acid transporter risk score as the instrumental variable; the results provided evidence that the SU levels were negatively correlated with TBIL. The Durbin-Hausman test provided further proof of the negative effect between exposure (SU) and outcomes (TBIL) with significant differences $(\mathrm{DHp}=0.002)$ in the adjusted analysis of TBIL. In addition, the individual SNPs were also analyzed in our study (Table 6). At $A B C G 2$ (rs1481012), negative beta and significant $\mathrm{DH} p$ 
Table 4: The OLS regression and the TLS regression analysis of SU against TBIL

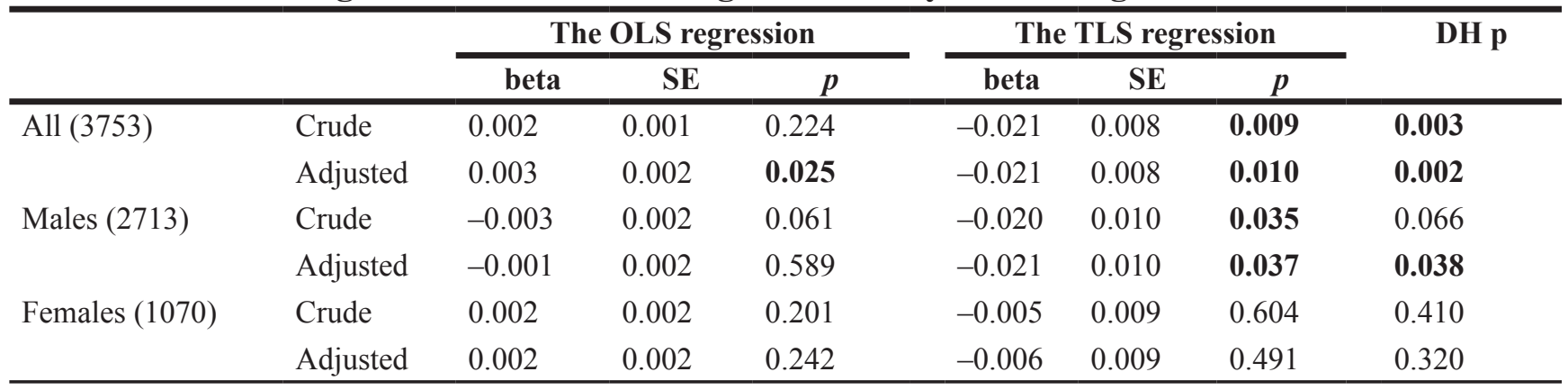

SE: Standard error; DH p: Durbin-Hausman $p$ value; Adjusted for age, gender (in All), BMI, BUN and FBG; DHp: DurbinHausman $p$ value; TLS: two-stage least square; OLS: ordinary least squares.

Table 5: The OLS regression and the TLS regression analysis of SU against TBIL in participants with high and normal SU

\begin{tabular}{lcccccccc}
\hline \multicolumn{1}{c}{ Participants } & \multicolumn{2}{c}{ The OLS regression } & \multicolumn{2}{c}{ The TLS regression } & DH p \\
& & beta & SE & $\boldsymbol{p}$ & beta & SE & $\boldsymbol{p}$ & \\
\hline Normal SU & & & & & & & & \\
All (2794) & Crude & 0.013 & 0.003 & $<\mathbf{0 . 0 0 1}$ & -0.047 & 0.029 & 0.109 & $\mathbf{0 . 0 2 5}$ \\
& Adjusted & 0.011 & 0.003 & $<\mathbf{0 . 0 0 1}$ & -0.047 & 0.028 & 0.092 & $\mathbf{0 . 0 0 2}$ \\
Males (1975) & Crude & 0.006 & 0.004 & 0.085 & -0.037 & 0.033 & 0.269 & 0.180 \\
& Adjusted & 0.007 & 0.004 & 0.063 & -0.037 & 0.033 & 0.264 & 0.170 \\
Females (819) & Crude & -0.001 & 0.004 & 0.849 & 0.018 & 0.025 & 0.477 & 0.450 \\
& Adjusted & -0.003 & 0.004 & 0.415 & 0.010 & 0.045 & 0.698 & 0.590 \\
Hyperuricemia & & & & & & & & \\
All (959) & Crude & -0.003 & 0.004 & 0.441 & -0.053 & 0.028 & $\mathbf{0 . 0 5 3}$ & $\mathbf{0 . 0 4 5}$ \\
& Adjusted & 0.001 & 0.004 & 0.854 & -0.053 & 0.028 & $\mathbf{0 . 0 2 7}$ & $\mathbf{0 . 0 1 0}$ \\
Males (733) & Crude & -0.007 & 0.005 & 0.141 & -0.046 & 0.030 & 0.124 & 0.170 \\
& Adjusted & -0.003 & 0.005 & 0.523 & -0.056 & 0.028 & $\mathbf{0 . 0 4 9}$ & $\mathbf{0 . 0 3 9}$ \\
Females (226) & Crude & 0.007 & 0.007 & 0.297 & -0.086 & 0.061 & 0.158 & 0.036 \\
& Adjusted & 0.010 & 0.007 & 0.147 & -0.107 & 0.090 & 0.240 & 0.050 \\
\hline
\end{tabular}

Adjusted for age, gender (in All), BMI, BUN and FBG; SE: Standard error; TLS: two-stage least square; OLS: ordinary least squares; DHp: Durbin-Hausman p value

values (beta $=-0.019, p=0.024, \mathrm{DHp}=0.006)$ were observed, suggesting the best evidence for a causal association between elevated SU and reduction of TBIL in this study. We also noted, from the F-statistics and $\mathrm{R}^{2}$, that $A B C G 2$ (rs2231137), SLC2A9 (rs16890979) and SLC17A1 (rs3799352), were weak instrumental variable and contributed very little to this analysis.

The assumption that elevated SU levels decrease the TBIL levels via induction of ROS and insulin resistance was considered. Tentative evidence was observed to support this hypothesis in previously published studies. Increasing concentrations of SU levels in the culture media of adipocytes induced a further increase in intracellular ROS production with activated NADPH [3]; others have reported that $\mathrm{SU}$ induces oxidative stress in adipocytes, vascular smooth muscle cells and human umbilical vein endothelial cell [31, 32]; Zhu et al. recently reported that hyperuricemia could increase ROS levels [33]. However, bilirubin showed powerful antioxidant properties [4-6] and could be oxidized to biliverbin by ROS, which can then protect cells from oxidative stress [7]. On the side, several studies has reported that high uric acid inhibits insulin signaling and induces insulin resistance [33, 34]. Interestingly, bilirubin could increase insulin sensitivity in published reported $[35,36]$. Taken together, these studies support the hypothesis that elevated SU levels can lead to increased ROS levels and induced insulin resistance, and resulting in reduction of TBIL levels. In addition, elevated SU may increase ROS levels in females, and 17ß-estradiol might inhibit expression of ROS [37-39]; therefore, the relationship between SU and TBIL was not significant in females. However, we found that the association between SU and TBIL is significant in 
Table 6: The TLS regression analysis of SU against TBIL using a single genetic variant as the instrument

\begin{tabular}{lccccccc}
\hline \multicolumn{1}{c}{ Gene (SNP) } & Subjects & F-statistic & $\mathbf{R}^{2}$ & beta & SE & $\boldsymbol{p}$ & DH p \\
\hline ABCG2 & All & 107.50 & 0.028 & -0.019 & 0.008 & $\mathbf{0 . 0 2 4}$ & $\mathbf{0 . 0 0 6}$ \\
(rs1481012) & Males & 78.33 & 0.028 & -0.026 & 0.011 & $\mathbf{0 . 0 1 9}$ & $\mathbf{0 . 0 1 7}$ \\
& Females & 46.67 & 0.043 & 0.003 & 0.009 & 0.779 & 0.990 \\
SLC2A9 & All & 10.13 & 0.003 & -0.133 & 0.058 & $\mathbf{0 . 0 2 3}$ & $<\mathbf{0 . 0 0 1}$ \\
(rs16890979) & Males & 5.72 & 0.002 & -0.186 & 0.112 & 0.097 & $<\mathbf{0 . 0 0 1}$ \\
& Females & 9.05 & 0.009 & -0.046 & 0.029 & 0.109 & $\mathbf{0 . 0 3 6}$ \\
ABCG2 & All & 35.51 & 0.009 & -0.010 & 0.017 & 0.429 & 0.300 \\
(rs2231137) & Males & 34.91 & 0.013 & -0.006 & 0.015 & 0.695 & 0.740 \\
& Females & 5.75 & 0.006 & -0.028 & 0.033 & 0.388 & 0.300 \\
SLC17A1 & All & 7.99 & 0.002 & -0.004 & 0.031 & 0.900 & 0.980 \\
(rs3799352) & Males & 7.48 & 0.003 & 0.018 & 0.035 & 0.609 & 0.580 \\
& Females & 1.24 & 0.001 & -0.056 & 0.101 & 0.576 & 0.440 \\
\hline
\end{tabular}

Adjusted for age, gender (in All), BMI, BUN and FBG; DHp: Durbin-Hausman $p$ value; TLS: two-stage least square.

males. Although the mechanism that underlies this genderspecific association is unclear, in this and other studies, higher levels of urate and bilirubin were observed in males than females $[40,41]$, which may explain the different statistical power of detection of urate-bilirubin associations in males vs. females. Our study provides an important insight in the understanding the potential mechanism of TBIL, elevated serum urate is a potential factor in reduction of total bilirubin. In addition, several published studies have been reported that bilirubin was a decrease risk factor in diabetes mellitus $[42,43]$ and cardiovascular disease $[44,45]$. Thus, it was quite important for physician to emphasize the risk of elevated SU and subjects should be strongly recommended to decrease high level of SU to avoid diabetes mellitus and cardiovascular disease.

Our study has a number of strengths. First, we used both the OLS and the Mendelian randomization approach, not merely a conventional observational method, to investigate exposure-outcome relationships and the potential relationship between SU and TBILrelated phenotypes in a Chinese population. This method allows a more precise conclusion of causality compared with traditional observational studies [19]. Second, the instrumental variable consisted of four SNPs, and the combined use of multiple variants as instruments increased the statistical power to test relationships between exposure and outcomes [22-24] and avoided the bias caused by weak instruments [25]. Finally, the uric acid transporter SNPs used were sufficient to represent the hereditary background of the Chinese population [46]. Nevertheless, this analysis has several limitations. Our study was conducted only with an older population, and further studies should be conducted in the general population. The data we analyzed was from a single, relatively small cohort, and further confirmation on our results in other cohorts from the Chinese population would be necessary. And our research merely focused on Chinese subjects, and studies using Mendelian randomization approach should be repeated in other populations.

In summary, although the relationship between SU and TBIL was likely to be very complex, our findings provide strong evidence that elevated SU levels is a potential factor in reduction of TBIL. To the best of our knowledge, this is the first research that investigates the relation between SU and TBIL using Mendelian randomization analysis, and it generated strong evidence to resolve the controversial association between $\mathrm{SU}$ and TBIL. Furthermore, our study provides an important insight in the understanding the potential mechanism of TBIL.

\section{MATERIALS AND METHODS}

\section{Study population}

The study population and methods of data collection were described previously [46]. This present study included 3,753 subjects (2,713 males and 1,040 females), and subjects who have been treated with urate-lowering drugs were excluded from this study. Characteristics of the participants, including age, gender, body mass index (BMI), fasting blood glucose (FBG), blood urea nitrogen (BUN), total biliverdin (TBIL) and serum urate (SU), were analyzed (Table 1). Subjects with high serum urate (> 417 $\mu \mathrm{mol} / \mathrm{L}$ ) were considered hyperuricemia patients [47]. This study was approved by the Ethical Committees of the School of Life Sciences of Fudan University and informed consent was obtained from each participant. 


\section{Instrumental variables}

For the uric acid transporter genetic risk score, SUincreasing alleles were selected based on our previous report [46]. In that study, in order to find the serum urate associated genes, 31 loci were tested in all individuals in Chinese. Then 11 loci in eight genes were shown to be associated with SU after multiple corrections. In summary, four single nucleotide polymorphisms (SNPs), which were reported to be associated with $\mathrm{SU}$ in literature, were selected as instrumental variables in our study: rs 1481012 in $A B C G 2$ (beta $\left.=27.555, p=3.50 \mathrm{E}-31, \mathrm{P}_{\mathrm{FDR}}=1.02 \mathrm{E}-29\right)$, rs2231137 in $A B C G 2$ (beta $=-16.945, p=2.33 \mathrm{E}-12, \mathrm{P}_{\mathrm{FDR}}=3.38 \mathrm{E}-11$ ), rs16890979 in $S L C 2 A 9$ (beta $=-24.654, p=0.014, \mathrm{P}_{\mathrm{FDR}}$ $=0.042$ ) and rs3799352 in SLC17A1 (beta $=-7.456, p=$ $5.35 \mathrm{E}-3, \mathrm{P}_{\mathrm{FDR}}=0.017$ ). We selected those four loci based on the following consideration: 1) The loci explain $4.2 \%$ of the variance of SU concentration, while approximately $7 \%$ for all known loci. 2) The loci have strong and unequivocal contributions to the concentration of SU based on our lab's previous study [46] and the reported genome-wide association studies [48-50]. However, regarding other loci, they show weak and ambiguous effects on SU and may cause controversial results. 3) Ethnicity have been proved to be a heterogeneous source for the association between genetic loci and SU. Some loci identified in EU and US populations did not affect serum urate levels in Chinese populations [51]. In our previous study, we had systematically studied the genetic basis of SU with all potential urate-related SNPs and found those four loci significantly influenced the concentrations of serum urate in this population [46]. 4) The loci corresponding genes encode urate transporters, which directly modify the SU concentrations. In order to get a more precise and powerful genetic risk score to explain the variance of SU, a weighted genetic risk score was calculated. An allele-counting genetic risk score was calculated. Based on the number of minor alleles that were associated with increased SU levels, each SNP was coded 0-2, and that number was multiplied by the corresponding beta value, and then the total genetic risk score was determined as the sum the four values. Most importantly, the instrumental variables selected must fulfill three assumptions [12, 26, 27]: the instrumental variables should be (1) robustly associated with the modifiable exposure of interest; (2) independent from confounding factors; (3) related to the outcome only via their association with the modifiable exposure.

\section{Statistical analysis}

First, the traditional observed relationship between SU and TBIL levels was analyzed using the ordinary least squares (OLS) regression. Second, the MRS was determined by conducting a two-stage least square (TLS) regression as previously described [52]. As shown above, the total genetic risk score calculated by four SNPs was selected as the instrumental variable in the TLS regression to assess the relationship between SU and TBIL levels. In the MRS, the F-statistic was examined from the first-stage regression and represents the strength between instrumental variables and exposures [53]; $\mathrm{R}^{2}$ represents the variance in $\mathrm{SU}$ as explained by the instrumental variables. Both the F-statistic and determination coefficient $\left(\mathrm{R}^{2}\right)$ were measured. According to the assumption of the Mendelian randomization approach, the F-statistic should be greater than ten [28], which would suggest that genetic instrumental variables were powerful enough to represent the level of SU. The relationship between the total genetic risk score and potential confounders was also assessed by linear regression. For the last step, the difference between the OLS regression and the TLS regression was measured by the DurbinHausman test [54]. If the $p$ value is significant, it suggests that the OLS regression cannot evaluate the relationship between exposure and outcome, and then the TLS regression is used for further analysis; while when the $p$ value is not significant, both the OLS and TLS regression can be used.

All analyses were carried by $\mathrm{R}$ (Version 3.2.2: www.r-project.org/). $P$ value less than 0.05 was determined statistical significance.

\section{CONFLICTS OF INTEREST}

The authors disclose no conflicts.

\section{FUNDING}

This study was supported by grants from the National Natural Science Foundation of China (31521003), Science and Technology Committee of Shanghai Municipality (11DJ1400102), International S\&T Cooperation Program of China (2013DFA30870), Ministry of Science and Technology (2011BAI09B00), 111 Project (B13016), and Program for 2012 Outstanding Medical Academic Leader for Hejian Zou. Computational support was provided by the High-End Computing Center located at Fudan University.

\section{REFERENCES}

1. Yang L, Dong Z, Ma Y, Pu W, Zhao D, He H, Ji H, Yang Y, Wang X, Xu X. Common UCP2 variants contribute to serum urate concentrations and the risk of hyperuricemia. Sci Rep. 2016; 6:27279.

2. Köttgen A, Albrecht E, Teumer A, Vitart V, Krumsiek J, Hundertmark C, Pistis G, Ruggiero D, O'Seaghdha CM, Haller T. Genome-wide association analyses identify 18 new loci associated with serum urate concentrations. Nat Genet. 2013; 45:145-54.

3. Sautin YY, Nakagawa T, Zharikov S, Johnson RJ. Adverse effects of the classic antioxidant uric acid in adipocytes: NADPH oxidase-mediated oxidative/nitrosative stress. Am J Physiol Cell Physiol. 2007; 293: C584-96. https://doi. org/10.1152/ajpcell.00600.2006. 
4. Duann P, Lianos EA. GEC-targeted HO-1 expression reduces proteinuria in glomerular immune injury. Am J Physiol Renal Physiol. 2009; 297:F629-38. https://doi. org/10.1152/ajprenal.00213.2009.

5. Abraham NG, Asija A, Drummond G, Peterson S. Heme oxygenase -1 gene therapy: recent advances and therapeutic applications. Curr Gene Ther. 2007; 7:89-108.

6. Baranano DE, Rao M, Ferris CD, Snyder SH. Biliverdin reductase: a major physiologic cytoprotectant. Proc Natl Acad Sci U S A. 2002; 99:16093-8. https://doi.org/10.1073/ pnas.252626999.

7. Schwertner HA, Vitek L. Gilbert syndrome, UGT1A1*28 allele, and cardiovascular disease risk: possible protective effects and therapeutic applications of bilirubin. Atherosclerosis. 2008; 198:1-11. https://doi.org/10.1016/j. atherosclerosis.2008.01.001.

8. Choi SH, Yun KE, Choi HJ. Relationships between serum total bilirubin levels and metabolic syndrome in Korean adults. Nutr Metab Cardiovasc Dis. 2013; 23:31-7. https:// doi.org/10.1016/j.numecd.2011.03.001.

9. Lee MJ, Jung CH, Kang YM, Hwang JY, Jang JE, Leem J, Park JY, Kim HK, Lee WJ. Serum bilirubin as a predictor of incident metabolic syndrome: a 4-year retrospective longitudinal study of 6205 initially healthy Korean men. Diabetes Metab. 2014; 40:305-9. https://doi.org/10.1016/j. diabet.2014.04.006.

10. Ishizaka N, Ishizaka $\mathrm{Y}$, Toda $\mathrm{E}$, Hashimoto $\mathrm{H}$, Nagai $\mathrm{R}$, Yamakado M. Higher serum uric acid is associated with increased arterial stiffness in Japanese individuals. Atherosclerosis. 2007; 192:131-7. https://doi.org/10.1016/j. atherosclerosis.2006.04.016.

11. Stojanov M, Stefanovic A, Dzingalasevic G, Ivanisevic J, Miljkovic M, Mandic-Radic S, Prostran M. Total bilirubin in young men and women: association with risk markers for cardiovascular diseases. Clin Biochem. 2013; 46:1516-9. https://doi.org/10.1016/j.clinbiochem.2013.06.020.

12. Lawlor DA, Harbord RM, Sterne JA, Timpson N, Davey Smith G. Mendelian randomization: using genes as instruments for making causal inferences in epidemiology. Stat Med. 2008; 27:1133-63. https://doi.org/10.1002/sim.3034.

13. Sumino H, Ichikawa S, Kanda T, Nakamura T, Sakamaki $\mathrm{T}$. Reduction of serum uric acid by hormone replacement therapy in postmenopausal women with hyperuricaemia. Lancet. 1999; 354: 650. https://doi.org/10.1016/s01406736(99)92381-4.

14. Bonora E, Targher G, Zenere MB, Saggiani F, Cacciatori V, Tosi F, Travia D, Zenti MG, Branzi P, Santi L, Muggeo M. Relationship of uric acid concentration to cardiovascular risk factors in young men. Role of obesity and central fat distribution. The Verona Young Men Atherosclerosis Risk Factors Study. Int J Obes Relat Metab Disord. 1996; 20:975-80.

15. McAdams DeMarco MA, Maynard JW, Baer AN, Gelber AC, Young JH, Alonso A, Coresh J. Diuretic use, increased serum urate levels, and risk of incident gout in a populationbased study of adults with hypertension: the Atherosclerosis
Risk in Communities cohort study. Arthritis Rheum. 2012; 64:121-9. https://doi.org/10.1002/art.33315.

16. Hingorani A, Humphries S. Nature's randomised trials. Lancet. 2005; 366:1906-8. https://doi.org/10.1016/s01406736(05)67767-7.

17. Davey Smith G, Ebrahim S. What can mendelian randomisation tell us about modifiable behavioural and environmental exposures? BMJ. 2005; 330:1076-9. https:// doi.org/10.1136/bmj.330.7499.1076.

18. McKeigue PM, Campbell H, Wild S, Vitart V, Hayward C, Rudan I, Wright AF, Wilson JF. Bayesian methods for instrumental variable analysis with genetic instruments ('Mendelian randomization'): example with urate transporter SLC2A9 as an instrumental variable for effect of urate levels on metabolic syndrome. Int J Epidemiol. 2010; 39:907-18. https://doi.org/10.1093/ije/dyp397.

19. Sheehan NA, Didelez V, Burton PR, Tobin MD. Mendelian randomisation and causal inference in observational epidemiology. PLoS Med. 2008; 5: e177. https://doi. org/10.1371/journal.pmed.0050177.

20. Smith GD, Ebrahim S. 'Mendelian randomization': can genetic epidemiology contribute to understanding environmental determinants of disease? Int J Epidemiol. 2003; 32:1-22.

21. Gupta V, Walia GK, Sachdeva MP. 'Mendelian randomization': an approach for exploring causal relations in epidemiology. Public Health. 2017; 145:113-9. https:// doi.org/10.1016/j.puhe.2016.12.033.

22. Palmer TM, Lawlor DA, Harbord RM, Sheehan NA, Tobias JH, Timpson NJ, Davey Smith G, Sterne JA. Using multiple genetic variants as instrumental variables for modifiable risk factors. Stat Methods Med Res. 2012; 21:223-42. https:// doi.org/10.1177/0962280210394459.

23. Burgess S, Butterworth A, Thompson SG. Mendelian randomization analysis with multiple genetic variants using summarized data. Genet Epidemiol. 2013; 37:658-65. https://doi.org/10.1002/gepi.21758.

24. Burgess S, Thompson SG. Use of allele scores as instrumental variables for Mendelian randomization. Int $\mathrm{J}$ Epidemiol. 2013; 42:1134-44. https://doi.org/10.1093/ije/ dyt093.

25. Davies NM, von Hinke Kessler Scholder S, Farbmacher H, Burgess S, Windmeijer F, Smith GD. The many weak instruments problem and Mendelian randomization. Stat Med. 2015; 34:454-68. https://doi.org/10.1002/sim.6358.

26. Glymour MM, Tchetgen Tchetgen EJ, Robins JM. Credible Mendelian randomization studies: approaches for evaluating the instrumental variable assumptions. Am J Epidemiol. 2012; 175:332-9. https://doi.org/10.1093/aje/kwr323.

27. Burgess S, Daniel RM, Butterworth AS, Thompson SG. Network Mendelian randomization: using genetic variants as instrumental variables to investigate mediation in causal pathways. Int J Epidemiol. 2015; 44:484-95. https://doi. org/10.1093/ije/dyu176. 
28. Burgess S, Thompson SG. Avoiding bias from weak instruments in Mendelian randomization studies. Int J Epidemiol. 2011; 40:755-64. https://doi.org/10.1093/ije/dyr036.

29. Hughes K, Flynn T, de Zoysa J, Dalbeth N, Merriman TR. Mendelian randomization analysis associates increased serum urate, due to genetic variation in uric acid transporters, with improved renal function. Kidney Int. 2014; 85:344-51. https://doi.org/10.1038/ki.2013.353.

30. Fetsch PA, Abati A, Litman T, Morisaki K, Honjo Y, Mittal K, Bates SE. Localization of the ABCG2 mitoxantrone resistanceassociated protein in normal tissues. Cancer Lett. 2006; 235:84-92. https://doi.org/10.1016/j.canlet.2005.04.024.

31. Yu MA, Sanchez-Lozada LG, Johnson RJ, Kang DH. Oxidative stress with an activation of the renin-angiotensin system in human vascular endothelial cells as a novel mechanism of uric acid-induced endothelial dysfunction. J Hypertens. 2010; 28:1234-42.

32. Corry DB, Eslami P, Yamamoto K, Nyby MD, Makino H, Tuck ML. Uric acid stimulates vascular smooth muscle cell proliferation and oxidative stress via the vascular reninangiotensin system. J Hypertens. 2008; 26:269-75. https:// doi.org/10.1097/HJH.0b013e3282f240bf.

33. Zhu Y, Hu Y, Huang T, Zhang Y, Li Z, Luo C, Luo Y, Yuan H, Hisatome I, Yamamoto T, Cheng J. High uric acid directly inhibits insulin signalling and induces insulin resistance. Biochem Biophys Res Commun. 2014; 447:70714. https://doi.org/10.1016/j.bbrc.2014.04.080.

34. Zhi L, Yuzhang Z, Tianliang H, Hisatome I, Yamamoto T, Jidong C. High Uric Acid Induces Insulin Resistance in Cardiomyocytes In Vitro and In Vivo. PLoS One. 2016; 11: e0147737. https://doi.org/10.1371/journal.pone.0147737.

35. Dong H, Huang H, Yun X, Kim DS, Yue Y, Wu H, Sutter A, Chavin KD, Otterbein LE, Adams DB, Kim YB, Wang $\mathrm{H}$. Bilirubin increases insulin sensitivity in leptin-receptor deficient and diet-induced obese mice through suppression of ER stress and chronic inflammation. Endocrinology. 2014; 155:818-28. https://doi.org/10.1210/en.2013-1667.

36. Liu J, Dong H, Zhang Y, Cao M, Song L, Pan Q, Bulmer A, Adams DB, Dong X, Wang H. Bilirubin Increases Insulin Sensitivity by Regulating Cholesterol Metabolism, Adipokines and PPARgamma Levels. Sci Rep. 2015; 5: 9886. https://doi.org/10.1038/srep09886.

37. Wagner AH, Schroeter MR, Hecker M. 17beta-estradiol inhibition of NADPH oxidase expression in human endothelial cells. FASEB J. 2001; 15:2121-30. https://doi. org/10.1096/fj.01-0123com.

38. Sumi D, Hayashi T, Matsui-Hirai H, Jacobs AT, Ignarro LJ, Iguchi A. 17beta-estradiol inhibits NADPH oxidase activity through the regulation of $\mathrm{p} 47$ phox mRNA and protein expression in THP-1 cells. Biochim Biophys Acta. 2003; 1640:113-8.

39. Arias-Loza PA, Muehlfelder M, Pelzer T. Estrogen and estrogen receptors in cardiovascular oxidative stress. Pflugers Arch. 2013; 465:739-46. https://doi.org/10.1007/ s00424-013-1247-7.
40. Zucker SD, Horn PS, Sherman KE. Serum bilirubin levels in the U.S. population: gender effect and inverse correlation with colorectal cancer. Hepatology. 2004; 40:827-35. https://doi.org/10.1002/hep.20407.

41. Suzuki S, Sagara K, Otsuka T, Matsuno S, Funada R, Uejima T, Oikawa Y, Koike A, Nagashima K, Kirigaya H, Yajima J, Sawada H, Aizawa T, et al. Gender-specific relationship between serum uric acid level and atrial fibrillation prevalence. Circ J. 2012; 76:607-11.

42. Cheriyath P, Gorrepati VS, Peters I, Nookala V, Murphy ME, Srouji N, Fischman D. High Total Bilirubin as a Protective Factor for Diabetes Mellitus: An Analysis of NHANES Data From 1999 - 2006. J Clin Med Res. 2010; 2:201-6. https://doi.org/10.4021/jocmr425w.

43. Abbasi A, Deetman PE, Corpeleijn E, Gansevoort RT, Gans RO, Hillege HL, van der Harst P, Stolk RP, Navis G, Alizadeh BZ, Bakker SJ. Bilirubin as a potential causal factor in type 2 diabetes risk: a Mendelian randomization study. Diabetes. 2015; 64:1459-69. https://doi.org/10.2337/ db14-0228.

44. Lin R, Wang Y, Wang Y, Fu W, Zhang D, Zheng H, Yu T, Wang Y, Shen M, Lei R, Wu H, Sun A, Zhang R, et al. Common variants of four bilirubin metabolism genes and their association with serum bilirubin and coronary artery disease in Chinese Han population. Pharmacogenet Genomics. 2009; 19:310-8. https://doi.org/10.1097/ FPC.0b013e328328f818.

45. Horsfall LJ, Nazareth I, Petersen I. Cardiovascular events as a function of serum bilirubin levels in a large, statintreated cohort. Circulation. 2012; 126:2556-64. https://doi. org/10.1161/circulationaha.112.114066.

46. Dong Z, Zhou J, Jiang S, Li Y, Zhao D, Yang C, Ma Y, Wang Y, He H, Ji H, Yang Y, Wang X, Xu X, et al. Effects of multiple genetic loci on the pathogenesis from serum urate to gout. Sci Rep. 2017; 7: 43614. https://doi.org/10.1038/ srep43614.

47. Khanna D, Fitzgerald JD, Khanna PP, Bae S, Singh MK, Neogi T, Pillinger MH, Merill J, Lee S, Prakash S, Kaldas M, Gogia M, Perez-Ruiz F, et al. 2012 American College of Rheumatology guidelines for management of gout. Part 1: systematic nonpharmacologic and pharmacologic therapeutic approaches to hyperuricemia. Arthritis Care Res (Hoboken). 2012; 64:1431-46. https://doi.org/10.1002/acr.21772.

48. Kottgen A, Albrecht E, Teumer A, Vitart V, Krumsiek J, Hundertmark C, Pistis G, Ruggiero D, O'Seaghdha CM, Haller T, Yang Q, Tanaka T, Johnson AD, et al. Genome-wide association analyses identify 18 new loci associated with serum urate concentrations. Nat Genet. 2013; 45:145-54. https://doi.org/10.1038/ng.2500.

49. Sulem P, Gudbjartsson DF, Walters GB, Helgadottir HT, Helgason A, Gudjonsson SA, Zanon C, Besenbacher S, Bjornsdottir G, Magnusson OT, Magnusson G, Hjartarson E, Saemundsdottir J, et al. Identification of low-frequency variants associated with gout and serum uric acid levels. Nat Genet. 2011; 43:1127-30. https://doi.org/10.1038/ng.972. 
50. Yang Q, Kottgen A, Dehghan A, Smith AV, Glazer NL, Chen MH, Chasman DI, Aspelund T, Eiriksdottir G, Harris TB, Launer L, Nalls M, Hernandez D, et al. Multiple genetic loci influence serum urate levels and their relationship with gout and cardiovascular disease risk factors. Circ Cardiovasc Genet. 2010; 3:523-30. https://doi.org/10.1161/ circgenetics.109.934455.

51. Yang B, Mo Z, Wu C, Yang H, Yang X, He Y, Gui L, Zhou L, Guo H, Zhang X, Yuan J, Dai X, Li J, et al. A genome-wide association study identifies common variants influencing serum uric acid concentrations in a Chinese population. BMC Med Genomics. 2014; 7:10. https://doi. org/10.1186/1755-8794-7-10.
52. Dalbeth N, Topless R, Flynn T, Cadzow M, Bolland MJ, Merriman TR. Mendelian randomization analysis to examine for a causal effect of urate on bone mineral density. J Bone Miner Res. 2015; 30:985-91. https://doi. org/10.1002/jbmr.2434.

53. Stock JH, Wright JH, Yogo M. A survey of weak instruments and weak identification in generalized method of moments. J Bus Econ Stat. 2002; 20:518-29.

54. Hausman JA. Specification tests in econometrics. Econometrica. 1978:1251-71. 